\title{
Socio-economic, demographic, lifestyle and health characteristics associated with consumption of fatty-sweetened and fatty-salted foods in middle-aged French adults
}

\author{
Caroline Méjean $^{1 *}$, Pauline Macouillard ${ }^{1}$, Katia Castetbon ${ }^{2}$, Emmanuelle Kesse-Guyot $^{1}$ and \\ Serge Hercberg ${ }^{1,2}$ \\ ${ }^{1}$ UREN (Unité de Recherche en Epidémiologie Nutritionnelle), UMR U557 Inserm/U1125 Inra/Cnam/Paris 13, CRNH IdF, \\ SMBH Paris 13, 74 rue Marcel Cachin, F-93017 Bobigny Cedex, France \\ ${ }^{2}$ USEN (Unité de Surveillance et d'Epidémiologie Nutritionnelle), Institut de Veille Sanitaire, Université Paris 13, 74 rue \\ Marcel Cachin, F-93017 Bobigny Cedex, France
}

(Received 17 February 2010 - Revised 14 September 2010 - Accepted 16 September 2010 - First published online 15 October 2010)

\begin{abstract}
Few studies have specifically focused on characteristics associated with consumption of combined fatty-salted and fatty-sweetened foods, whereas their identification could be useful for defining effective public health measures. The aim of the present study was to investigate the association between demographic, socio-economic, lifestyle and health characteristics and consumption of these types of food in a general sample of French adults. Dietary intake was assessed using a minimum of six $24 \mathrm{~h}$ dietary records collected over a 2-year period in 6240 subjects aged 35-60 years who participated in the Supplémentation en VItamines Minéraux et AntioXydants cohort study. Associations of individual characteristics with high and intermediate consumption of fatty-sweetened and fatty-salted foods were assessed using multivariate polytomic logistic regression models. Risk of moderate or high consumption of fatty-salted foods decreased with increasing age. Current smokers, drinkers, individuals with overweight and with hypertension were more likely to consume moderate or high amounts of such foods. Risk of moderate or high consumption of fatty-sweetened foods decreased with increasing age. Women, individuals living as a couple, moderate drinkers and persons with low or medium physical activity level were more likely to consume moderate or high amounts of such foods. Lower educated subjects, current smokers, heavy drinkers and individuals with severe hypertriacylglycerolaemia were less likely to have moderate or high consumption. Consumption of fatty-sweetened and fatty-salted foods varied according to demographic, lifestyle and health characteristics. Common unhealthy behaviours such as smoking, low physical activity and alcohol drinking, associated with high consumption of these food groups, may help to effectively target public health efforts.
\end{abstract}

Key words: Fatty-sweetened foods: Fatty-salted foods: Lifestyle: Socio-economic factors: Demographic factors

Dietary components such as fat, added sugar and $\mathrm{Na}$ are important nutritional factors involved in the risk of chronic diseases $^{(1,2)}$. Indeed, a high consumption of foods containing added sugar, notably beverages, has been linked to the risk of obesity, diabetes and heart disease ${ }^{(3,4)}$. Moreover, overconsumption of high-fat products may play a role in the development of obesity, CVD and colorectal cancer $^{(5,6)}$. Finally, excessive salt consumption may contribute to hypertension and gastric cancers, at least in some sensitive populations ${ }^{(7,8)}$.

Most public health programmes worldwide target nutritional recommendations, which include limitations on fat, salt and sugar products ${ }^{(2)}$. Identification and characterisation of population subgroups whose consumption of these products is too high may help public health policymakers to disseminate messages and take actions which could improve the dietary behaviour of the population in a more focused and efficient manner.

Several studies have investigated intakes of fat, added sugar and $\mathrm{Na}$ and their association with demographic, socio-economic, lifestyle and health characteristics ${ }^{(9-11)}$, but few studies have specifically focused on the characteristics of combined fatty-salted or fatty-sweetened food consumers $^{(12-14)}$. Nevertheless, elucidation of the combination of fatty and salted foods or fatty and sweetened products would be useful, since combining salt or sugar with fat may promote consumption of fat by increasing palatability ${ }^{(15,16)}$. The inference is that increased

Abbreviation: SU.VI.MAX, Supplémentation en VItamines Minéraux et AntioXydants. 
palatability could lead to overconsumption, thus creating an additional risk factor for obesity and health-related problems ${ }^{(17)}$. Elsewhere, available studies were mainly interested in demographic and socio-economic profiles of consumption of these foods, but rarely assessed associations with lifestyle ${ }^{(12)}$.

The aim of the present study was to investigate the association between demographic, socio-economic, lifestyle and health characteristics and consumption of fattysalted foods, on the one hand, and fatty-sweetened foods, on the other hand, in a large sample of middleaged subjects participating in a French cohort.

\section{Methods}

\section{Study population}

We used data from the 'Supplémentation en VItamines Minéraux et AntioXydants' (SU.VI.MAX) cohort, a large group of middle-aged adults recruited throughout mainland France. The design, methods and rationale of the present study have been described elsewhere ${ }^{(18)}$. The SU.VI.MAX study was initially designed as a randomised, double-blind, placebo-controlled primary prevention trial to test the efficacy of daily supplementation with antioxidant vitamins and minerals at nutritional doses in reducing the incidence of IHD, cancers and overall mortality ${ }^{(18)}$. A total of 7876 women aged 35-60 years and 5141 men aged 45-60 years were included in 1994-5 for an initially planned follow-up of 8 years. They consisted of volunteers recruited in 1994 through a large national media campaign via television, radio and newspapers. To be eligible, volunteers had to return a completed screening questionnaire. Further eligibility criteria included: absence of disease likely to hinder active participation or threaten 5-year survival; acceptance of the possibility of being given a placebo and of constraints of participation; no previous regular supplementation with any of the vitamins or minerals in the supplement provided; absence of extremist beliefs or behaviour regarding diet. The present study was conducted according to the guidelines laid down in the Declaration of Helsinki, and all procedures involving human subjects were approved by the Ethical Committee for Studies with Human Subjects at the Paris-Cochin Hospital (CCPPRB no. 706) and the Commission Nationale Informatique et Liberté (CNIL no. 334641). Written informed consent was obtained from all subjects ${ }^{(19)}$.

\section{Assessment of fatty-sweetened and fatty-salted food consumption}

Dietary data collection. Participants were invited to provide a $24 \mathrm{~h}$ dietary record every 2 months. These records were randomly distributed for 2 weekend days and 4 weekdays/year, so that each day of the week and all seasons were covered to account for individual variability in intake. Dietary data were collected using the Minitel Telematic Network, a small terminal that was widely used in France at the beginning of the study (1994-6). At enrolment, participants received a tiny central processing unit specifically developed for the study, containing specialised software that allowed subjects to fill out the computerised dietary record off-line and to transmit data during brief telephone connections. Conversational facilities of the software and an instruction manual for codification of foods, distributed to the participants at enrolment, guided them during completion of the records. A large choice of food and drink (990 food items) was displayed for each of three meals (breakfast, lunch and dinner) and for four other food intake occurrences. For each food or drink mentioned, subjects were asked to indicate the portion size eaten. They were helped by the instruction manual which included photographs of more than 250 foods (corresponding to 990 generic foods) presented in three different portion sizes. Along with the two intermediate and two extreme quantities, there were seven choices of amounts. Edible amounts corresponding to each food had been previously validated in a pilot study ${ }^{(19)}$. The nutritional values of the diet were estimated using a published French food composition table ${ }^{(20)}$. All dietary records with outlying values $(<418$ or $>25080 \mathrm{~kJ} / \mathrm{d})$ were reviewed by dietitians. They checked and corrected data-entry mistakes in declared quantities and errors due to data transfer (record transferred into several pieces). When no error was identified, the aberrant record was deleted.

\section{Definition of fatty-sweetened and fatty-salted food groups}

Since there exists no internationally acknowledged recommendation for the classification of food groups, we defined two food groups taking into account their nutritional content in fat, sugar and salt: 'fatty-sweetened' foods and 'fatty-salted' foods. The fatty-sweetened food group included high-fat yogurt and sweetened cream desserts, chocolate products, croissant-like pastries and other sweetened pastries, cakes and biscuits. For these food groups, the nutrient standards were lipids $\geq 15 \mathrm{~g} / 100 \mathrm{~g}$ and added sugars $\geq 10 \mathrm{~g} / 100 \mathrm{~g}$. The fatty-salted food group was composed of processed meats, cheese, appetisers, sandwiches, hamburgers, pizzas and savoury pastries and sauces. The nutrient standards for these food groups were lipids $\geq 15 \mathrm{~g} / 100 \mathrm{~g}$ and $\mathrm{Na} \geq 500 \mathrm{mg} / 100 \mathrm{~g}$.

\section{Scoring}

To construct scores of fatty-sweetened and fatty-salted food consumption, we defined servings from commonly used portion sizes on the French market for each food. One point was attributed for each food belonging to the fatty-salted group or the fatty-sweetened group when the subject ate one serving. When the subject consumed less than or more than the defined servings, we assigned 
points proportionally to the quantity consumed. The number of points was summed up for each subject to obtain a total score for each fatty group divided by the number of days recorded, in order to standardise expression of the scores.

\section{Assessment of demographic, socio-economic and lifestyle characteristics}

At enrolment, subjects were asked to fill in a self-administered questionnaire to provide demographic and socioeconomic information. Based on the eight initial categories, education level was recoded into three categories according to the highest level completed (primary, secondary or university level). The official French classification was used to classify subjects into occupational categories according to their self-reported occupation or most recent employment if they were retired or unemployed. Occupational category was recoded into four categories (from nine initial categories) and a fifth included retired persons, unemployed individuals and persons who had never worked. Marital status was coded into single and married or living with a partner. Smoking status was categorised into three categories: current smokers, former smokers and never smokers. Physical activity level was assessed using a non-validated method, via a single question. The subjects were asked to report whether they regularly practised physical activity and, if so, whether they practised the equivalent of at least $1 \mathrm{~h}$ of walking/d. Responses were coded into three categories (irregular or no physical activity, $<1 \mathrm{~h}$ of walking/d, $\geq 1 \mathrm{~h}$ of walking/d) ${ }^{(21)}$. The subjects who stated that they were 'irregular' in engaging in physical activities were considered as having low physical activity $(<30 \mathrm{~min} / \mathrm{d}$ of equivalent walking). Those who indicated that they walked more than $1 \mathrm{~h} / \mathrm{d}$ were considered as having high physical activity. The mean daily alcohol intake was estimated from at least six $24 \mathrm{~h}$ dietary records. For each record, the day of recording was randomly selected over a 2-week period to provide dispersion of survey days and thus to cover differences between days of the week, particularly between weekend days and weekdays. The mean daily alcohol intake was expressed as grams of alcohol consumed per day, corresponding to the sum of alcohol consumed per day from wine, beer, spirits and cider. According to French recommendations ${ }^{(22)}$, male drinkers were categorised as non-drinkers $(0 \mathrm{~g}$ alcohol/d), moderate ( $>0$ and $\leq 30 \mathrm{~g}$ alcohol/d) or heavy drinkers $(>30 \mathrm{~g}$ alcohol $/ \mathrm{d})$, and females as non-drinkers ( $0 \mathrm{~g}$ alcohol/d), moderate $(>0$ and $\leq 20 \mathrm{~g}$ alcohol/d) or heavy drinkers $(>20 \mathrm{~g}$ alcohol/d).

\section{Clinical examination and laboratory methods}

A clinical examination was conducted in 1995-6. Weight and height were measured using standardised procedures $^{(23)}$. BMI $\left(\mathrm{kg} / \mathrm{m}^{2}\right)$ was calculated, and overweight was defined as a BMI of $25 \mathrm{~kg} / \mathrm{m}^{2(24)}$. Blood pressure was measured on each arm using a standard Hg sphygmomanometer in subjects who had been lying down for at least $10 \mathrm{~min}$. The mean of these two measurements was used for analyses. Hypertension was defined using WHO/International Society of Hypertension cut-offs ${ }^{(25)}$ : systolic blood pressure $\geq 140 \mathrm{mmHg}$ and/or diastolic blood pressure $\geq 90$ $\mathrm{mmHg}$ and/or anti-hypertensive medication.

Blood samples were obtained at baseline after $12 \mathrm{~h}$ fasting, and all biochemical measurements were centralised. Serum total cholesterol and TAG levels were measured using an enzymatic method (Bayer Diagnostics, Tarrytown, NY, USA), and fasting glucose by a glucose-oxidase method (Bayer Diagnostics). According to French health authorities ${ }^{(26,27)}$, limits were used for elevated levels of total cholesterol $(5 \cdot 2<$ moderate $<7 \mathrm{mmol} / \mathrm{l}$; severe $\geq 7 \mathrm{mmol} / \mathrm{l})$, elevated levels of TAG $(1.7<$ moderate $<2.3 \mathrm{mmol} / \mathrm{l}$; severe $\geq 2.3 \mathrm{mmol} / \mathrm{l}$ ) and elevated levels of glycaemia (glycaemia $\geq 7 \mathrm{mmol} / \mathrm{l}$ ). Individuals with hypercholesterolaemia, hypertriacylglycerolaemia or diabetes also included those who declared that they were being treated for each of these cardiovascular risks, respectively.

\section{Statistical analyses}

The present analyses focused on subjects aged 35-60 years who had completed at least six 24 h dietary records (some subjects had filled in more than six records, with the median number of $24 \mathrm{~h}$ records being 11) during the first 2 years of follow-up (1994-6). Given that missing data for demographic, socio-economic, lifestyle characteristics and variables represented less than $3 \%$ of the sample, we deleted subjects with no available data for these variables $^{(28)}$. For blood pressure, $14 \%$ of the sample had missing values. Therefore, regression-based imputation taking into account sex, age and weight was performed. To explore bias in selection of the sample, we compared individuals from the entire SU.VI.MAX population with those who had completed at least six dietary records and those included in the final sample (without missing data), using a $\chi^{2}$ goodness-of-fit test for categorical variables and one-sample $t$ test for continuous variables.

Tertiles of score were estimated in order to define 'low', 'intermediate' and 'elevated' consumption of fattysweetened foods, on the one hand, and fatty-salted foods, on the other hand. Logistic regression analysis was used to assess demographic, socio-economic, lifestyle and health factors related to elevated and intermediate consumption of fatty-sweetened and fatty-salty foods (polytomic model ${ }^{(29)}$, common reference $=$ low consumption). Univariate logistic regressions were performed by calculating OR and 95\% CI to determine the strength of the association between levels of consumption and each explanatory variable (see afore-mentioned description; all were tested). Since we hypothesised that the sex would 
probably modify the association of the other explanatory variables with the likelihood of consumption levels, interaction terms were also examined. The significance level for interactions was set at $0 \cdot 20$.

Only explanatory variables associated with food group consumption at the $0 \cdot 20$ significance level were retained for inclusion in the initial multivariate model. Subsequently, using stepwise backward elimination, multivariate logistic regression models were constructed. All models were adjusted for energy intake and for sex. Variables and interaction terms were removed from the model one by one using $P>0.10$ for exclusion. Variables whose exclusion from the model caused large fluctuations in OR $(>10 \%)$, as well as variables whose exclusion gave rise to significant likelihood-ratio tests $(P<0.05)$, were re-entered into the model. Significance tests were two-sided, with a $P$ value set at $<0 \cdot 05$. All statistical analyses were performed using Statistical Analysis Systems (version 9.1; SAS Institute, Inc., Cary, NC, USA).

\section{Results}

Characteristics of consumers of fatty-salted and fattysweetened foods

Of the 13017 participants in the SU.VI.MAX study, 6633 participants provided at least six valid $24 \mathrm{~h}$ dietary records during the first 2 years of follow-up. Next, the present analysis was performed on a subsample of 6240 subjects who had no missing values for covariates. In the sample which included individuals with at least six dietary records ( $n$ 6633) and in the final sample used for analyses here ( $n$ 6240), men and hypertensive individuals were proportionally more numerous, and single persons and current smokers were less numerous, than in the entire cohort ( $n$ 13 017) (Table 1). Significant differences in occupational categories and in alcohol consumption categories were also found.

Demographic, socio-economic, lifestyle and health characteristics of the total sample and of consumers of the fatty-salted and fatty-sweetened foods are presented in Table 2. Total intakes of energy $(P<0.0001)$, lipids $(P<0.0001), \mathrm{Na}(P<0.0001)$ and added sugar $(P<0.0001)$ increased according to the levels of consumption of fattysalted foods (Table 1). In addition, the higher the increase in consumption of fatty-sweetened foods, the greater the increase in intakes of total energy $(P<0.0001)$, lipids $(P<0.0001)$, added sugar $(P<0.0001)$ and $\mathrm{Na}(P<0.0001)$.

\section{Characteristics of consumption of fatty-salted foods}

Except for the variable concerning hypercholesterolaemia, all demographic, socio-economic, lifestyle and health variables tested here were selected from univariate analyses (Table 2) for introduction into the multivariate model. In addition, significant interactions of sex with education level $(P=0.003)$, occupational category $(P=0 \cdot 06)$ and physical activity level $(P=0.06)$ were found and therefore introduced into the multivariate model. After a stepwise backward elimination procedure, sex, marital status, education level, occupational category, physical activity level, variables relating to diabetes and hypertriacylglycerolaemia and interactions terms were excluded.

In the final model, age was inversely associated with the risk of being a medium or high consumer (Table 3). Moderate and heavy alcohol drinkers were more likely to have moderate or high consumption of fatty-salted foods than abstainers. In addition, compared with never smokers, current smokers were more likely to be medium or high consumers of these foods. Overweight subjects were more likely to eat higher quantities of fatty-salted products than individuals with normal or low body mass. Finally, individuals with hypertension were more likely to be medium or high consumers of fatty-salted products than those without hypertension.

Results of the stratified analyses by sex did not greatly differ from those in the model which included both sexes (detailed results not shown). Nevertheless, the association between fatty-salted food consumption and alcohol consumption was significant in both sexes, but was slightly less strong in men than in women. In addition, estimates of an association of consumption of fatty-salted foods with hypertension and smoking status were higher in men than in women.

\section{Characteristics of consumption of fatty-sweetened foods}

All demographic, socio-economic, lifestyle and health variables were selected from univariate analyses (Table 2) for introduction into the multivariate model. Moreover, significant interactions of sex with hypertension $(P=0.06)$ and smoking status $(P=0.06)$ were selected to be introduced into the multivariate model. After a stepwise backward selection procedure, occupational category, weight status, variables relating to diabetes, hypercholesterolaemia and hypertension and interaction terms were excluded.

In the final model (Table 4), the risk of moderate or high consumption of fatty-sweetened foods decreased when age increased. In addition, women and individuals who lived as a couple were more likely to consume such foods in moderate or high amounts. Compared with individuals with a high education level, individuals with a low education level were less likely to have moderate or high consumption of fatty-sweetened foods, and those with a medium education level were less likely to be high consumers. Moreover, current smokers were less likely to be moderate consumers than never smokers. Compared with abstainers, moderate alcohol drinkers were more likely to have moderate consumption of fatty-sweetened foods, whereas heavy alcohol drinkers were less likely to be high consumers of these foods. Subjects with low and medium physical activity levels were more likely to consume higher amounts of 
Table 1. Comparisons of demographic, socio-economic, lifestyle and health characteristics between the Supplémentation en Vltamines Minéraux et AntioXydants (SU.VI.MAX) population and individuals with at least six dietary records and those without missing data

(Mean values and standard deviations or percentages)

\begin{tabular}{|c|c|c|c|c|c|}
\hline Variables & $\begin{array}{l}\text { SU.VI.MAX } \\
\text { population } \\
(n 13017)\end{array}$ & $\begin{array}{l}\text { Sample including individuals } \\
\text { with at least six dietary } \\
\text { records ( } n 6633)\end{array}$ & $P$ & $\begin{array}{l}\text { Final sample for } \\
\text { analysis ( } n \text { 6240) }\end{array}$ & $P$ \\
\hline Age (years) & & & 0.0002 & & 0.0002 \\
\hline Mean & $48 \cdot 4$ & $48 \cdot 7$ & & $48 \cdot 7$ & \\
\hline SD & $6 \cdot 3$ & 6.4 & & $6 \cdot 4$ & \\
\hline \multicolumn{6}{|l|}{ Sex } \\
\hline Men & 39.5 & $42 \cdot 4$ & 0.0004 & $42 \cdot 4$ & $<0.0001$ \\
\hline Women & $60 \cdot 5$ & $57 \cdot 6$ & & $57 \cdot 6$ & \\
\hline \multicolumn{6}{|l|}{ Occupational category } \\
\hline Self-employed and farmers & 5.9 & 4.7 & $<0.0001$ & 4.7 & $<0.0001$ \\
\hline $\begin{array}{l}\text { Managerial staff and intermediate } \\
\text { professions }\end{array}$ & $54 \cdot 8$ & $55 \cdot 1$ & & $55 \cdot 3$ & \\
\hline Manual workers and employees & $16 \cdot 6$ & $16 \cdot 0$ & & $16 \cdot 0$ & \\
\hline Unemployed, never employed and retired & $22 \cdot 7$ & $24 \cdot 2$ & & 24.0 & \\
\hline \multicolumn{6}{|l|}{ Education level } \\
\hline Elementary school & $21 \cdot 3$ & $20 \cdot 7$ & 0.40 & $20 \cdot 5$ & 0.25 \\
\hline Secondary school & $38 \cdot 1$ & $38 \cdot 6$ & & $38 \cdot 6$ & \\
\hline University or equivalent & $40 \cdot 6$ & $40 \cdot 7$ & & $40 \cdot 9$ & \\
\hline \multicolumn{6}{|l|}{ Marital status } \\
\hline Single & $17 \cdot 7$ & $14 \cdot 8$ & $<0.0001$ & $14 \cdot 8$ & $<0.0001$ \\
\hline Married or living with a partner & $82 \cdot 3$ & $85 \cdot 2$ & & $85 \cdot 2$ & \\
\hline \multicolumn{6}{|l|}{ Physical activity level } \\
\hline Low $(<30 \mathrm{~min} / \mathrm{d})$ & $25 \cdot 3$ & 24.8 & 0.17 & 24.9 & 0.14 \\
\hline Medium $(<1 \mathrm{~h}$ and $\geq 30 \mathrm{~min} / \mathrm{d})$ & 31.3 & $30 \cdot 6$ & & 30.5 & \\
\hline High $(\geq 1 \mathrm{~h} / \mathrm{d})$ & 43.4 & $44 \cdot 6$ & & $44 \cdot 6$ & \\
\hline \multicolumn{6}{|l|}{ Smoking status } \\
\hline Never smoker & $47 \cdot 0$ & $48 \cdot 2$ & $<0.0001$ & $48 \cdot 0$ & $<0.0001$ \\
\hline Former smoker & $37 \cdot 0$ & $38 \cdot 3$ & & 38.6 & \\
\hline Current smoker & $16 \cdot 0$ & 13.5 & & $13 \cdot 4$ & \\
\hline \multicolumn{6}{|l|}{ Alcohol consumption ( $g$ ethanol) } \\
\hline Non-drinker & $21 \cdot 2$ & 11.5 & $<0.0001$ & 13.9 & $<0.0001$ \\
\hline $\begin{array}{l}\text { Moderate drinker }(\mathrm{M}:>0 \text { and } \leq 30 \mathrm{~g} / \mathrm{d} \text {; } \\
\mathrm{F}:>0 \text { and } \leq 20 \mathrm{~g} / \mathrm{d})\end{array}$ & $45 \cdot 6$ & $52 \cdot 3$ & & $51 \cdot 4$ & \\
\hline Heavy drinker $(\mathrm{M}:>30 \mathrm{~g} / \mathrm{d}$; F: $>20 \mathrm{~g} / \mathrm{d})$ & $33 \cdot 2$ & $36 \cdot 2$ & & 34.7 & \\
\hline \multicolumn{6}{|l|}{ Health status } \\
\hline Overweight $\left(\mathrm{BMI} \geq 25 \mathrm{~kg} / \mathrm{m}^{2}\right)$ & $35 \cdot 9$ & 34.4 & 0.09 & $34 \cdot 1$ & 0.11 \\
\hline $\begin{array}{l}\text { Hypertension }(\mathrm{SBP} \geq 140 \mathrm{mmHg} \\
\text { and/or DBP } \geq 90 \mathrm{mmHg} \\
\text { and/or medication) }\end{array}$ & $23 \cdot 6$ & $26 \cdot 7$ & $<0.0001$ & $26 \cdot 7$ & $<0.0001$ \\
\hline $\begin{array}{l}\text { Diabetes (glycaemia } \geq 7 \mathrm{mmol} / \mathrm{l} \\
\text { and/or medication) }\end{array}$ & $4 \cdot 3$ & $4 \cdot 0$ & 0.25 & $4 \cdot 0$ & 0.25 \\
\hline $\begin{array}{l}\text { Moderated hypertriacylglycerolaemia } \\
(1.7 \mathrm{mmol} / \mathrm{l}<\text { triacylglycerolaemia } \\
<2.3 \mathrm{mmol} / \mathrm{l} \text { and/or medication })\end{array}$ & $11 \cdot 9$ & $12 \cdot 1$ & 0.05 & $12 \cdot 0$ & 0.05 \\
\hline Severe hypertriacylglycerolaemia $(\geq 2.3 \mathrm{mmol} / \mathrm{l})$ & $6 \cdot 3$ & $5 \cdot 2$ & & $5 \cdot 2$ & \\
\hline $\begin{array}{l}\text { Moderate hypercholesterolaemia } \\
(5.2 \mathrm{mmol} / / \text { < cholesterolaemia } \\
<7 \mathrm{mmol} / \mathrm{l} \text { and/or medication) }\end{array}$ & $62 \cdot 4$ & 62.5 & 0.83 & $62 \cdot \overline{6}$ & 0.96 \\
\hline Severe hypercholesterolaemia ( $\geq 7 \mathrm{mmol} / \mathrm{l})$ & $16 \cdot 4$ & $16 \cdot 5$ & & $16 \cdot 4$ & \\
\hline
\end{tabular}

M, male; F, female; SBP, systolic blood pressure; DBP, diastolic blood pressure.

fatty-sweetened foods. Finally, individuals with severe hypertriacylglycerolaemia were less likely to consume such foods in moderate or high amounts than those without hypertriacylglycerolaemia.

Results of the stratified analyses by sex did not differ from those of the model which included both sexes, except for smoking status (detailed results not shown). Male current smokers and former smokers were less likely to have moderate consumption of fatty-sweetened foods than male never smokers, whereas female former smokers were more likely to consume such foods in high amounts compared with female never smokers.

\section{Discussion}

The present study, which specifically focused on the consumption of fatty-salted and fatty-sweetened foods, emphasises the importance of considering fat, salt and added sugar not just as single components of the diet, but rather as components whose interactions could contribute to high consumption. Thus, the present results may help to identify high-risk profiles among consumers of these unhealthy food groups, which would be useful for setting up nutritional guidelines in public health. The findings from our research show that consumption of 
Table 2. Dietary, demographic, socio-economic, lifestyle and health characteristics of consumers of fatty-salted and fatty-sweetened foods

(Mean values and standard deviations or percentages)

\begin{tabular}{|c|c|c|c|c|c|c|c|c|c|}
\hline \multirow[b]{2}{*}{ Variables } & \multirow[b]{2}{*}{ Total sample } & \multicolumn{3}{|c|}{ Fatty-salted food consumption } & \multirow[b]{2}{*}{$P$} & \multicolumn{3}{|c|}{ Fatty-sweetened food consumption } & \multirow[b]{2}{*}{$P$} \\
\hline & & Low & Intermediate & High & & Low & Intermediate & High & \\
\hline \multicolumn{10}{|l|}{ Total energy intake (kJ/d) } \\
\hline Mean & $8870 \cdot 1$ & 7263.4 & 8695.6 & 10683.8 & \multirow[t]{2}{*}{$<0.0001$} & $8200 \cdot 6$ & $8750 \cdot 8$ & $9642 \cdot 8$ & \multirow[t]{2}{*}{$<0.0001$} \\
\hline SD & 2466.0 & 1158.5 & $1925 \cdot 0$ & 2278.6 & & 2429.2 & $2296 \cdot 2$ & 2463.1 & \\
\hline \multicolumn{10}{|l|}{ Lipid intake (g/d) } \\
\hline Mean & 88.9 & $70 \cdot 2$ & 87.0 & $110 \cdot 0$ & $<0.0001$ & 80.9 & 87.6 & $98 \cdot 2$ & \multirow[t]{2}{*}{$<0.0001$} \\
\hline SD & 26.5 & 19.1 & $19 \cdot 0$ & 24.1 & & $25 \cdot 7$ & 23.9 & 26.9 & \\
\hline \multicolumn{10}{|l|}{ Na intake (mg/d) } \\
\hline Mean & 3478.0 & $2796 \cdot 8$ & $3428 \cdot 3$ & 4222.4 & $<0.0001$ & 3348.9 & 3455.8 & $3625 \cdot 9$ & \multirow[t]{2}{*}{$<0.0001$} \\
\hline SD & $1184 \cdot 3$ & $938 \cdot 3$ & 963.4 & $1175 \cdot 4$ & & $1223 \cdot 2$ & 1128.5 & $1187 \cdot 7$ & \\
\hline \multicolumn{10}{|l|}{ Added sugar intake (g/d) } \\
\hline Mean & 40.8 & $35 \cdot 2$ & 41.4 & 45.9 & $<0.0001$ & 32.1 & 39.0 & $51 \cdot 1$ & \multirow[t]{2}{*}{$<0.0001$} \\
\hline SD & 21.4 & $19 \cdot 2$ & 20.8 & 22.9 & & $19 \cdot 0$ & 18.4 & $22 \cdot 8$ & \\
\hline \multicolumn{10}{|l|}{ Age (years) } \\
\hline Mean & 48.7 & 48.2 & $48 \cdot 2$ & 49.6 & $<0.0001$ & 49.5 & 48.7 & 47.9 & \multirow[t]{2}{*}{$<0.0001$} \\
\hline SD & 6.4 & 6.5 & 6.4 & 6.0 & & $6 \cdot 2$ & 6.4 & 6.4 & \\
\hline \multicolumn{10}{|l|}{ Sex } \\
\hline Men & $42 \cdot 4$ & $23 \cdot 2$ & 38.8 & $65 \cdot 6$ & \multirow[t]{2}{*}{$<0.0001$} & 43.9 & 39.9 & 43.5 & \multirow[t]{2}{*}{0.02} \\
\hline Women & 57.6 & 76.8 & $61 \cdot 2$ & 34.4 & & $56 \cdot 1$ & 60.1 & $56 \cdot 6$ & \\
\hline \multicolumn{10}{|l|}{ Occupational category } \\
\hline Self-employed and farmers & 4.7 & 3.8 & 5.0 & $5 \cdot 3$ & $<0.0001$ & 4.7 & 4.4 & $5 \cdot 0$ & 0.003 \\
\hline $\begin{array}{l}\text { Managerial staff and intermediate } \\
\text { professions }\end{array}$ & $55 \cdot 3$ & $51 \cdot 6$ & $56 \cdot 8$ & 57.6 & & 51.9 & $56 \cdot 0$ & $58 \cdot 0$ & \\
\hline Manual workers and employees & $16 \cdot 0$ & 18.8 & 14.6 & 14.5 & & 17.7 & $15 \cdot 3$ & 14.9 & \\
\hline $\begin{array}{l}\text { Unemployed, never employed and } \\
\text { retired }\end{array}$ & 24.0 & $25 \cdot 8$ & $23 \cdot 6$ & 22.6 & & $25 \cdot 8$ & $24 \cdot 3$ & $22 \cdot 0$ & \\
\hline Education level & & & & & & & & & \\
\hline Elementary school & 20.5 & 20.7 & 18.9 & 21.9 & 0.006 & $24 \cdot 0$ & $20 \cdot 1$ & 17.5 & $<0.0001$ \\
\hline Secondary school & 38.6 & $40 \cdot 2$ & 37.2 & 38.4 & & 39.7 & 39.2 & 37.0 & \\
\hline University or equivalent & 40.9 & 39.1 & 43.9 & 39.7 & & 36.4 & 40.8 & $45 \cdot 4$ & \\
\hline Marital status & & & & & & & & & \\
\hline Single & $14 \cdot 8$ & $17 \cdot 0$ & $14 \cdot 3$ & $13 \cdot 0$ & 0.001 & 13.5 & 14.9 & 15.9 & 0.09 \\
\hline Married or living with a partner & $85 \cdot 2$ & 83.1 & 85.7 & 87.0 & & 86.6 & 85.1 & 84.1 & \\
\hline Physical activity level & & & & & & & & & \\
\hline $\operatorname{Low}(<30 \mathrm{~min} / \mathrm{d})$ & 24.9 & 25.5 & 24.7 & 24.5 & 0.02 & 24.7 & 25.4 & $24 \cdot 6$ & 0.02 \\
\hline Medium $(<1 \mathrm{~h}$ and $\geq 30 \mathrm{~min} / \mathrm{d})$ & 30.5 & 32.1 & $31 \cdot 2$ & 28.3 & & $28 \cdot 2$ & 30.7 & 32.7 & \\
\hline High $(\geq 1 \mathrm{~h} / \mathrm{d})$ & 44.6 & 42.4 & 44.2 & $47 \cdot 2$ & & $47 \cdot 2$ & 43.9 & $42 \cdot 8$ & \\
\hline Smoking status & & & & & & & & & \\
\hline Never smoker & 48.0 & 54.7 & 49.5 & 39.7 & $<0.0001$ & 47.5 & $51 \cdot 0$ & $45 \cdot 3$ & 0.003 \\
\hline Former smoker & 38.6 & 31.0 & 38.4 & 46.6 & & 38.2 & 36.6 & $41 \cdot 1$ & \\
\hline Current smoker & 13.4 & 14.4 & $12 \cdot 1$ & 13.7 & & 14.4 & $12 \cdot 3$ & 13.6 & \\
\hline Alcohol consumption ( $\mathrm{g}$ ethanol) & & & & & & & & & \\
\hline Non-drinker & 13.9 & 22.7 & $12 \cdot 2$ & 6.8 & $<0.0001$ & $16 \cdot 4$ & $12 \cdot 4$ & $13 \cdot 2$ & 0.0005 \\
\hline $\begin{array}{l}\text { Moderate drinker (M: }>0 \text { and } \\
\leq 30 \mathrm{~g} / \mathrm{d} ; \mathrm{F}:>0 \text { and } \leq 20 \mathrm{~g} / \mathrm{d} \text { ) }\end{array}$ & 51.4 & $57 \cdot 3$ & 56.5 & $40 \cdot 2$ & & $48 \cdot 2$ & 52.7 & 53.2 & \\
\hline $\begin{array}{l}\text { Heavy drinker (M: > } 30 \mathrm{~g} / \mathrm{d} \text {; } \\
\mathrm{F}:>20 \mathrm{~g} / \mathrm{d})\end{array}$ & 34.7 & $20 \cdot 0$ & $31 \cdot 3$ & $52 \cdot 9$ & & 35.5 & 34.9 & 33.6 & \\
\hline Health status & & & & & & & & & \\
\hline Overweight (BMI $\geq 25 \mathrm{~kg} / \mathrm{m}^{2}$ ) & 34.1 & 28.6 & $30 \cdot 3$ & 43.5 & $<0.0001$ & 38.6 & 32.6 & $31 \cdot 3$ & $<0.0001$ \\
\hline $\begin{array}{l}\text { Hypertension } \\
\text { (SBP } \geq 140 \mathrm{mmHg} \text { and/or DBP }\end{array}$ & 26.7 & 21.6 & $26 \cdot 2$ & 32.6 & $<0.0001$ & 29.5 & 25.8 & $25 \cdot 0$ & 0.003 \\
\hline
\end{tabular}


fatty-salted and fatty-sweetened foods is independently related to various characteristics, such as demographic, lifestyle and health factors, in this large national sample of French middle-aged women and men. Besides, the direction of the association between lifestyle characteristics (smoking and alcohol) and high consumption was opposite according to whether food was fatty-salted or fatty-sweetened.

Consumption of fatty-sweetened foods varied according to several demographic factors, whereas consumption of fatty-salted foods was related only to age. The intake of fatty-salted and fatty-sweetened foods decreased with age in our population. As discussed previously ${ }^{(30)}$, the intake of energy-dense sweets and fast foods may decline with age due to physiological and psychological changes. Given that the present result concerned a population aged 35-60 years, these changes may occur during this period. In agreement with the literature ${ }^{(13,31)}$, consumption of fatty-sweetened foods also varied with sex, showing a higher intake among women. A review on consumption of foods by men and women highlighted the fact that consumption of sweet foods such as cakes, biscuits, puddings and chocolate is tacitly treated by men and women as a marker of femininity in many cultures ${ }^{(31)}$. In addition, the present results showed that individuals who were married or living as a couple were higher consumers of fattysweetened foods. In general, marriage and living as a couple are related to healthy eating habits ${ }^{(32,33)}$. However, some studies also showed that people who lived as a couple ate more energy-dense foods such as sweets and desserts than single people $e^{(12,34,35)}$. Finally, the present results highlight the fact that low- and middle-educated individuals were lower consumers of fatty-sweetened foods; this result is not consistent with the previous literature, which showed a higher intake of high-fat dairy products, cakes and desserts in subjects having a lower education level ${ }^{(14,36)}$. However, fatty-sweetened foods, such as sweet cream desserts, chocolate products and pastries, in the period under study (1994-6), could represent up-to-date and sophisticated products, particularly in urban areas. After the later large-scale nutritional information campaigns, perception of these products changed and fruits were preferred to them among the more highly educated individuals.

The present study showed that a high consumption of fatty-salted or fatty-sweetened foods were associated with other unhealthy lifestyles, including low physical activity, smoking habits and alcohol consumption. Our findings were in agreement with previous studies, which showed that unhealthy dietary patterns (higher intake of fat, higher intake of high-fat dairy products, cakes, chocolate, etc.) and unhealthy physical activity patterns were correlated behaviours ${ }^{(37)}$. Regular physical activity seemed to be integrated into a 'healthy' lifestyle along with dietary aspects $^{(38-40)}$. 
Table 3. Multivariate polytomic logistic analysis of the association between demographic, socio-economic, lifestyle and health characteristics and levels of fatty-salted food consumption*

(Odds ratios and $95 \%$ confidence intervals)

\begin{tabular}{|c|c|c|c|c|c|c|c|}
\hline & \multicolumn{3}{|c|}{ Medium $v$. low consumers } & \multicolumn{3}{|c|}{ High $v$. low consumers } & \multirow[b]{2}{*}{ Global $P \ddagger$} \\
\hline & OR & $95 \% \mathrm{Cl} \dagger$ & $P$ & OR & $95 \% \mathrm{Cl} \dagger$ & $P$ & \\
\hline Age (years) & 0.98 & $0.97,0.99$ & $<0.0001$ & 0.98 & $0.96,0.99$ & 0.0004 & $<0.0001$ \\
\hline \multicolumn{8}{|l|}{ Sex } \\
\hline Men & 1.00 & & & 1.00 & & & \\
\hline Women & 0.96 & $0.81,1.14$ & 0.67 & 0.90 & $0.74,1.10$ & 0.33 & 0.61 \\
\hline \multicolumn{8}{|l|}{ Smoking } \\
\hline Never smoker & 1.00 & & & 1.00 & & & \\
\hline Former smoker & 0.85 & $0.69,1.04$ & 0.08 & 1.07 & $0.84,1.35$ & 0.58 & 0.006 \\
\hline Current smoker & $1 \cdot 15$ & $1.00,1.33$ & 0.004 & 1.29 & $1.09,1.53$ & 0.01 & \\
\hline \multicolumn{8}{|l|}{ Alcohol consumption } \\
\hline Non-drinker & 1.00 & & & 1.00 & & & \\
\hline $\begin{array}{l}\text { Moderate drinker }(\mathrm{M}:>0 \text { and } \leq 30 \mathrm{~g} \\
\text { alcohol } / \mathrm{d} ; \mathrm{F}:>0 \text { and } \leq 20 \mathrm{~g} \text { alcohol } / \mathrm{d} \text { ) }\end{array}$ & 1.46 & $1 \cdot 22,1 \cdot 76$ & 0.004 & 1.54 & $1.21,1.98$ & 0.14 & $<0.0001$ \\
\hline $\begin{array}{l}\text { Heavy drinker }(\mathrm{M}:>30 \mathrm{~g} \text { alcohol } / \mathrm{d} \text {; } \\
\mathrm{F}:>20 \mathrm{~g} \text { alcohol } / \mathrm{d})\end{array}$ & 1.45 & $1 \cdot 16,1 \cdot 81$ & 0.04 & $1 \cdot 86$ & $1.41,2.45$ & $<0.0001$ & \\
\hline \multicolumn{8}{|l|}{ Body mass status } \\
\hline Normal $\left(\mathrm{BMI}<25 \mathrm{~kg} / \mathrm{m}^{2}\right)$ & 1.00 & & & 1.00 & & & \\
\hline Overweight $\left(\mathrm{BMI} \geq 25 \mathrm{~kg} / \mathrm{m}^{2}\right)$ & 1.06 & $0.91,1.23$ & 0.46 & 1.57 & $1.32,1.86$ & $<0.0001$ & $<0.0001$ \\
\hline \multicolumn{8}{|l|}{ Hypertension } \\
\hline Normal & 1.00 & & & 1.00 & & & \\
\hline $\begin{array}{l}\text { Hypertensive }(\mathrm{SBP} \geq 140 \mathrm{mmHg} \text { and/or } \\
\text { DBP } \geq 90 \mathrm{mmHg} \text { and/or anti-hypertensive } \\
\text { medication) }\end{array}$ & $1 \cdot 27$ & $1.08,1.49$ & 0.004 & $1 \cdot 33$ & $1 \cdot 11,1 \cdot 60$ & 0.002 & 0.002 \\
\hline
\end{tabular}

M, male; F, female; SBP, systolic blood pressure; DBP, diastolic blood pressure.

${ }^{*}$ Variables included in the model were age, sex, smoking status, alcohol consumption, BMI, presence of hypertension and total daily energy intake (in kJ/d, continuous variable).

$\dagger$ Determined by logistic regression analyses.

¥ Global $P$ value represented the overall significance of each variable, retained in the final model (type 3 analysis of effects).

The present study also showed that, compared with individuals who never smoked, current smokers consumed more fatty-salted and less fatty-sweetened foods. While the literature showed that smoking is strongly associated with unhealthy dietary patterns ${ }^{(41)}$, several studies have also found that current smokers had lower intakes of fatty-sweetened foods ${ }^{(42,43)}$. Indeed, behavioural research showed that cigarette smoking is accompanied by decreased consumption of sweet-tasting high-energy foods ${ }^{(44)}$. The association between smoking and fatty-salted foods in the literature was more difficult to evaluate $^{(45,46)}$. However, sensory research showed that smoking reduces the perception of a salty taste due to long-term-induced sensory deficits ${ }^{(47)}$. Smokers might need to consume more salty products to attain taste sensations of the same magnitude as non-smokers.

The findings showing that alcohol drinkers were higher consumers of fatty-salted foods but lower consumers of fatty-sweetened foods were in concordance with previous studies ${ }^{(39,40)}$. Colditz et al. $^{(39)}$ evoked the possibility that sweet food items and alcohol may be competitors in the diet. For fatty-salted foods, one experimental study showed that participants who drank higher doses of alcohol ate more high-fat salty food items, reflecting changes in taste perception of salty foods following a dose of alcohol $^{(48)}$. There may exist a behavioural explanation for combined consumption of alcohol and these foods.
Indeed, fatty-salted foods such as appetisers and cheese are typically eaten with alcoholic beverages in France.

Causal inferences regarding a possible association between consumption and health characteristics must be viewed with caution, due to the cross-sectional design of the present study. On the one hand, individuals with severe hypertriacylglycerolaemia may change their diet after learning about their disease, by reducing their intake of unhealthy food, and therefore were lower consumers of fatty-sweetened foods than those with normal triacylglycerolaemia values even after adjustment. One the other hand, the present results showed that overweight and/or hypertensive subjects were higher consumers of fattysalted products. Certain dietary behaviours, risk factors of these diseases, may be more difficult to modify. Thus, this finding encourages reinforcing dietary management in the treatment of these diseases ${ }^{(2)}$.

Interpretation of the present results must take into account the characteristics of the study. The subjects were participants in a nutritional intervention study and may therefore have a healthier lifestyle than the general population. Thus, caution is needed when interpreting and generalising results to the adult population in France. However, the present study included a large sample of subjects from a free-living population; moreover, the characteristics of participants in the SU.VI.MAX study were close to those of the national population in terms of geographical density and socio-economic status ${ }^{(18)}$. 
Table 4. Multivariate polytomic logistic analysis of the association between demographic, socio-economic, lifestyle and health characteristics and levels of fatty-sweetened food consumption*

(Odds ratios and 95\% confidence intervals)

\begin{tabular}{|c|c|c|c|c|c|c|c|}
\hline & \multicolumn{3}{|c|}{ Medium $v$. low consumers } & \multicolumn{3}{|c|}{ High $v$. low consumers } & \multirow[b]{2}{*}{ Global $P \ddagger$} \\
\hline & OR & $95 \% \mathrm{Cl} \dagger$ & $P$ & OR & $95 \% \mathrm{Cl}+$ & $P$ & \\
\hline Age (years) & 0.99 & $0.98,1.00$ & 0.008 & 0.97 & $0.96,0.98$ & $<0.0001$ & $<0.0001$ \\
\hline \multicolumn{8}{|l|}{ Sex } \\
\hline Men & 1.00 & & & 1.00 & & & \multirow[t]{2}{*}{$<0.0001$} \\
\hline Women & 1.51 & $1 \cdot 27,1 \cdot 78$ & $<0.0001$ & 1.76 & $1 \cdot 47,2 \cdot 11$ & $<0.0001$ & \\
\hline \multicolumn{8}{|l|}{ Marital status } \\
\hline Single & 1.00 & & & 1.00 & & & \multirow[t]{2}{*}{0.03} \\
\hline Married or living with a partner & $1 \cdot 15$ & $0.96,1.37$ & 0.13 & 1.28 & $1.06,1.54$ & 0.009 & \\
\hline \multicolumn{8}{|l|}{ Education level } \\
\hline University or equivalent & 1.00 & & & 1.00 & & & \multirow[t]{3}{*}{0.004} \\
\hline Secondary school & 0.92 & $0.80,1.05$ & 0.22 & 0.83 & $0.72,0.96$ & 0.01 & \\
\hline Elementary school & 0.83 & $0.70,0.98$ & 0.03 & 0.71 & $0.59,0.85$ & 0.0002 & \\
\hline \multicolumn{8}{|l|}{ Smoking status } \\
\hline Never smoker & 1.00 & & & 1.00 & & & \multirow[t]{3}{*}{0.006} \\
\hline Former smoker & 0.90 & $0.78,1.03$ & 0.12 & $1 \cdot 12$ & $0.97,1.30$ & 0.13 & \\
\hline Present smoker & 0.79 & $0.65,0.96$ & 0.02 & 1.01 & $0.82,1.24$ & 0.93 & \\
\hline \multicolumn{8}{|l|}{ Alcohol consumption } \\
\hline Non-drinker & 1.00 & & & 1.00 & & & \multirow[t]{3}{*}{$<0.0001$} \\
\hline $\begin{array}{l}\text { Moderate drinker }(\mathrm{M}:>0 \text { and } \\
\leq 30 \mathrm{~g} \text { alcohol } / \mathrm{d} ; \mathrm{F}:>0 \text { and } \leq 20 \mathrm{~g} \text { alcohol } / \mathrm{d})\end{array}$ & $1 \cdot 33$ & $1 \cdot 11,1 \cdot 61$ & 0.003 & 1.07 & $0.88,1.30$ & 0.50 & \\
\hline $\begin{array}{l}\text { Heavy drinker }(\mathrm{M}:>30 \mathrm{~g} \text { alcohol/d; } \\
\mathrm{F}:>20 \mathrm{~g} \text { alcohol/d) }\end{array}$ & $1 \cdot 13$ & $0.91,1.41$ & 0.27 & 0.61 & $0.49,0.77$ & $<0.0001$ & \\
\hline \multicolumn{8}{|l|}{ Physical activity level } \\
\hline High ( $\geq 1 \mathrm{~h}$ of walking/d) & 1.00 & & & 1.00 & & & \multirow[t]{3}{*}{0.04} \\
\hline Medium ( $<1 \mathrm{~h}$ and $\geq 30 \mathrm{~min}$ of walking/d) & 1.12 & $0.97,1.30$ & 0.12 & 1.26 & $1.08,1.47$ & 0.004 & \\
\hline Low $(<30 \mathrm{~min}$ of walking/d) & $1 \cdot 14$ & $0.97,1.33$ & 0.11 & $1 \cdot 19$ & $1.01,1.41$ & 0.04 & \\
\hline \multicolumn{8}{|l|}{ Triacylglycerol } \\
\hline Normal & & & & & & & \multirow[t]{3}{*}{0.02} \\
\hline $\begin{array}{l}\text { Moderate hypertriacylglycerolaemia } \\
(1.7 \mathrm{mmol} / \mathrm{l}<\text { triacylglycerolaemia }<2.3 \mathrm{mmol} / \mathrm{l} \\
\text { and/or medication) }\end{array}$ & 0.92 & $0.76,1 \cdot 12$ & 0.41 & 0.83 & $0.68,1.03$ & 0.09 & \\
\hline Severe hypertriacylglycerolaemia ( $\geq 2.3 \mathrm{mmol} / \mathrm{l})$ & 0.70 & $0.53,0.93$ & 0.01 & 0.66 & $0.49,0.89$ & 0.006 & \\
\hline
\end{tabular}

M, male; F, female; SBP, systolic blood pressure; DBP, diastolic blood pressure.

*Variables included in the model were age, sex, smoking status, alcohol consumption, BMI, presence of hypertension and total daily energy intake (in kJ/d, continuous variable).

$\dagger$ Determined by logistic regression analyses.

$¥$ Global $P$ value represented the overall significance of each variable, retained in the final model (type 3 analysis of effects).

Another limitation was the use of the food record as a method for dietary assessment. A major disadvantage of the dietary record method is that it leads to bias due to selection of participants in the study and to reliability in dietary assessment ${ }^{(49)}$. First, the requirement for frequent dietary recording may have discouraged responders from participating. Indeed, numerous participants were excluded from the present analysis because they filled fewer than six computerised $24 \mathrm{~h}$ dietary records. The differences that were found in socio-economic, demographic and lifestyle profiles between the total sample of the SU.VI.MAX study and those individuals could have caused bias in the studied associations because of potential underestimation of fatty-sweetened and fatty-salted food consumption in men, single people, current smokers and alcohol drinkers. Nevertheless, selection of at least six dietary records is also a strength of the present study, since it enabled reliable dietary estimation and limited misclassification by decreasing intra-individual variations ${ }^{(50)}$. In addition, the knowledge that food requires recording and the demanding task of doing it might alter the dietary behaviour that the tool was intended to measure ${ }^{(51)}$. The combined effects of under-recording of foods considered to be unhealthy and the impact of the recording process on dietary behaviour changes, leading to under-eating, may have caused the participants to underestimate usual dietary habits. Indeed, the results of comparisons of dietary intake between the first day of recording and later days highlighted a significant difference in lipid intake, indicating slight under-reporting of fatty foods. In order to classify foods as fatty-sweetened or fatty-salted, we applied arbitrary thresholds based on nutritional content in fat, sugar and salt. However, the strength of the present study lies in the use of food groups rather than a nutrient approach, which may be more appropriate for analysing the association of dietary behaviour with lifestyle. Another strength of the present study was the use of a large number of covariates to assess potential socio-economic, demographic, lifestyle and health characteristics of consumers of fattysalted and fatty-sweetened foods.

In conclusion, the sensory properties of combined fattysweetened or fatty-salted products contribute to eating 
pleasure and might therefore lead to overconsumption, identified as a risk factor in obesity and related health problems ${ }^{(15-17)}$. In this respect, the present study provides original and useful information on the characteristics of consumers of these food groups, simultaneously taking into account demographic, socio-economic, lifestyle and health characteristics. In particular, the present results, which identify common correlates of unhealthy behaviours such as smoking, low physical activity and alcohol drinking and high consumption of fatty-sweetened or fatty-salted foods, may help to more accurately target public health efforts. Campaigns to improve diet and increase physical activity, along with encouraging a reduction in cigarette smoking and alcohol consumption, might be useful for improving the nutritional status of populations.

\section{Acknowledgements}

This study was supported by the French National Research Agency (Agence Nationale de la Recherche) in the context of the 2008 Programme de Recherche 'Alimentation et Industries Alimentaires' (ANR-08-ALIA-006). None of the authors had a conflict of interest. C. M. designed the study, supervised the statistical analysis, interpreted data and drafted the manuscript. P. M. performed data management and statistical analyses and participated in the drafting of the manuscript. E. K.-G. and K. C. were involved in the conception and design of the study and interpretation of the data, and helped to draft the manuscript. S. H. designed and coordinated the study cohort and supervised the study. All authors critically reviewed the paper and approved the final version submitted for publication. We thank all the scientists who helped carry out the SU.VI.MAX study, the teams that assisted in fieldwork and the dedicated and conscientious volunteers who participated in this cohort.

\section{References}

1. American Institute for Cancer Research/World Cancer Research Fund (2007) Food, Nutrition, Physical Activity and the Prevention of Cancer: A Global Perspective. Washington, DC: American Institute for Cancer Research.

2. World Health Organization (2003) Diet, Nutrition and the Prevention of Chronic Diseases. Joint WHO/FAO Expert Consultation. WHO Technical Report Series no. 916. Geneva: WHO.

3. Fung TT, Malik V, Rexrode KM, et al. (2009) Sweetened beverage consumption and risk of coronary heart disease in women. Am J Clin Nutr 89, 1037-1042.

4. Vartanian LR, Schwartz MB \& Brownell KD (2007) Effects of soft drink consumption on nutrition and health: a systematic review and meta-analysis. Am J Public Health 97, 667-675.

5. Getz GS \& Reardon CA (2007) Nutrition and cardiovascular disease. Arterioscler Thromb Vasc Biol 27, 2499-2506.

6. Santarelli RL, Pierre F \& Corpet DE (2008) Processed meat and colorectal cancer: a review of epidemiologic and experimental evidence. Nutr Cancer 60, 131-144.
7. Gibbs CR, Lip GY \& Beevers DG (2000) Salt and cardiovascular disease: clinical and epidemiological evidence. J Cardiovasc Risk 7, 9-13.

8. Law M (2000) Salt, blood pressure and cardiovascular diseases. J Cardiovasc Risk 7, 5-8.

9. Leclercq C \& Ferro-Luzzi A (1991) Total and domestic consumption of salt and their determinants in three regions of Italy. Eur J Clin Nutr 45, 151-159.

10. Mattisson I, Wirfalt E, Gullberg B, et al. (2001) Fat intake is more strongly associated with lifestyle factors than with socio-economic characteristics, regardless of energy adjustment approach. Eur J Clin Nutr 55, 452-461.

11. Thompson FE, McNeel TS, Dowling EC, et al. (2009) Interrelationships of added sugars intake, socioeconomic status, and race/ethnicity in adults in the United States: National Health Interview Survey, 2005. J Am Diet Assoc 109, 1376-1383.

12. Deshmukh-Taskar P, Nicklas TA, Yang SJ, et al. (2007) Does food group consumption vary by differences in socioeconomic, demographic, and lifestyle factors in young adults? The Bogalusa Heart Study. J Am Diet Assoc 107, 223-234.

13. Macdiarmid JI, Vail A, Cade JE, et al. (1998) The sugar-fat relationship revisited: differences in consumption between men and women of varying BMI. Int J Obes Relat Metab Disord 22, 1053-1061.

14. Sanchez-Villegas A, Martinez JA, Prattala R, et al. (2003) A systematic review of socioeconomic differences in food habits in Europe: consumption of cheese and milk. Eur J Clin Nutr 57, 917-929.

15. Emmett PM \& Heaton KW (1995) Is extrinsic sugar a vehicle for dietary fat? Lancet 345, 1537-1540.

16. Kanarek RB, Ryu M \& Przypek J (1995) Preferences for foods with varying levels of salt and fat differ as a function of dietary restraint and exercise but not menstrual cycle. Physiol Behav 57, 821-826.

17. Yeomans MR, Blundell JE \& Leshem M (2004) Palatability: response to nutritional need or need-free stimulation of appetite? Br J Nutr 92, Suppl. 1, S3-S14.

18. Hercberg S, Preziosi P, Briancon S, et al. (1998) A primary prevention trial using nutritional doses of antioxidant vitamins and minerals in cardiovascular diseases and cancers in a general population: the SU.VI.MAX study - design, methods, and participant characteristics. SUpplementation en VItamines et Mineraux AntioXydants. Control Clin Trials 19, 336-351.

19. Le Moullec N, Deheeger M, Preziosi P, et al. (1996) Validation du manuel photos utilisé pour l'enquête alimentaire de l'étude SU.VI.MAX (Validation of the photo manual used for the collection of dietary data in the SU.VI.MAX Study). Cab Nutr Diet 31, 158-164.

20. Hercberg S (co-ordinator) (2005) Table de composition SU.VI.MAX des aliments (Food Composition Table of the SU.VI.MAX Study). Paris: Les éditions INSERM/Economica.

21. Kesse-Guyot E, Bertrais S, Peneau S, et al. (2009) Dietary patterns and their sociodemographic and behavioural correlates in French middle-aged adults from the SU.VI.MAX cohort. Eur J Clin Nutr 63, 521-528.

22. Hercberg S, Chat-Yung S \& Chauliac M (2008) The French National Nutrition and Health Program: 2001-2006-2010. Int J Public Health 53, 68-77.

23. Lohman TG, Roche AF \& Martorell R (1988) Anthropometric Standardization Reference Manual. Champaign, IL: Human Kinetics.

24. World Health Organization (1995) Physical Status: The Use and Interpretation of Anthropometry. Report of a WHO Expert Committee. WHO Technical Report Series no. 854. Geneva: WHO. 
25. Whitworth JA (2003) World Health Organization (WHO)/ International Society of Hypertension (ISH) statement on management of hypertension. J Hypertens 21, 1983-1992.

26. Agence française de sécurité sanitaire des produits de santé (Afssaps) (2005) Prise en charge thérapeutique du patient dyslipidémique (Therapeutic Management of Dyslipidemic Patients). Afssaps: Seine Saint Denis.

27. Haute autorité de santé (2006) Traitement médicamenteux du diabète de type 2 - Actualisation 2006 - Recommandations (Drug Treatment of Type 2 Diabetes - Update 2006 Recommendations). Paris: HAS.

28. Fox-Wasylyshyn SM \& El Masri MM (2005) Handling missing data in self-report measures. Res Nurs Health $\mathbf{2 8}$, 488-495.

29. Hosmer DW \& Lemeshow S (2000) Applied Logistic Regression. New York: Wiley Series in Probability and Mathematical Statistics.

30. Drewnowski A \& Shultz JM (2001) Impact of aging on eating behaviors, food choices, nutrition, and health status. J Nutr Health Aging 5, 75-79.

31. O'Doherty JK \& Holm L (1999) Preferences, quantities and concerns: socio-cultural perspectives on the gendered consumption of foods. Eur J Clin Nutr 53, 351-359.

32. Roos E, Lahelma E, Virtanen M, et al. (1998) Gender, socioeconomic status and family status as determinants of food behaviour. Soc Sci Med 46, 1519-1529.

33. Yannakoulia M, Panagiotakos D, Pitsavos C, et al. (2008) Eating patterns may mediate the association between marital status, body mass index, and blood cholesterol levels in apparently healthy men and women from the ATTICA study. Soc Sci Med 66, 2230-2239.

34. Eng PM, Kawachi I, Fitzmaurice G, et al. (2005) Effects of marital transitions on changes in dietary and other health behaviours in US male health professionals. I Epidemiol Community Health 59, 56-62.

35. Lee S, Cho E, Grodstein F, et al. (2005) Effects of marital transitions on changes in dietary and other health behaviours in US women. Int J Epidemiol 34, 69-78.

36. Fraser GE, Welch A, Luben R, et al. (2000) The effect of age, sex, and education on food consumption of a middle-aged English cohort-EPIC in East Anglia. Prev Med 30, 26-34.

37. Gillman MW, Pinto BM, Tennstedt S, et al. (2001) Relationships of physical activity with dietary behaviors among adults. Prev Med 32, 295-301.
38. Reedy J, Haines PS \& Campbell MK (2005) The influence of health behavior clusters on dietary change. Prev Med $\mathbf{4 1}$, $268-275$.

39. Colditz GA, Giovannucci E, Rimm EB, et al. (1991) Alcohol intake in relation to diet and obesity in women and men. Am J Clin Nutr 54, 49-55.

40. Kesse E, Clavel-Chapelon F, Slimani N, et al. (2001) Do eating habits differ according to alcohol consumption? Results of a study of the French cohort of the European Prospective Investigation into Cancer and Nutrition (E3N-EPIC). Am J Clin Nutr 74, 322-327.

41. Dallongeville J, Marecaux N, Fruchart JC, et al. (1998) Cigarette smoking is associated with unhealthy patterns of nutrient intake: a meta-analysis. J Nutr 128, 1450-1457.

42. Palaniappan U, Jacobs SL, O'Loughlin J, et al. (2001) Fruit and vegetable consumption is lower and saturated fat intake is higher among Canadians reporting smoking. $J$ Nutr 131, 1952-1958.

43. Whichelow MJ, Erzinclioglu SW \& Cox BD (1991) A comparison of the diets of non-smokers and smokers. BrJ Addict $\mathbf{8 6}$, $71-81$.

44. Grunberg NE (1982) The effects of nicotine and cigarette smoking on food consumption and taste preferences. Addict Behav 7, 317-331.

45. Ma J, Hampl JS \& Betts NM (2000) Antioxidant intakes and smoking status: data from the continuing survey of food intakes by individuals 1994-1996. Am J Clin Nutr 71, 774-780.

46. Touvier M, Niravong M, Volatier JL, et al. (2009) Dietary patterns associated with vitamin/mineral supplement use and smoking among women of the E3N-EPIC cohort. Eur $J$ Clin Nutr 63, 39-47.

47. Frye RE, Schwartz BS \& Doty RL (1990) Dose-related effects of cigarette smoking on olfactory function. JAMA 263, 1233-1236.

48. Caton SJ, Ball M, Ahern A, et al. (2004) Dose-dependent effects of alcohol on appetite and food intake. Physiol Behav 81, 51-58.

49. Thompson FE, Subar AF, Loria CM, et al. (2010) Need for technological innovation in dietary assessment. J Am Diet Assoc 110, 48-51.

50. Sempos CT, Johnson NE, Smith EL, et al. (1985) Effects of intraindividual and interindividual variation in repeated dietary records. Am J Epidemiol 121, 120-130.

51. Rebro SM, Patterson RE, Kristal AR, et al. (1998) The effect of keeping food records on eating patterns. J Am Diet Assoc 98, 1163-1165. 